\title{
TIME SYMMETRY AND ASYMMETRY IN QUANTUM MECHANICS AND QUANTUM COSMOLOGY
}

\author{
Murray Gell-Mann ${ }^{1}$ and James B. Hartle ${ }^{2}$, \\ ${ }^{1}$ Lauritsen Laboratory \\ California Institute of Technology, Pasadena, CA 91125 \\ ${ }^{2}$ Department of Physics \\ University of California, Santa Barbara, CA 93106-9530
}

(Dated: February 3, 2008)

*Electronic address: hartle@physics.ucsb.edu 


\section{INTRODUCTION}

The disparity between the time symmetry of the fundamental laws of physics and the time asymmetries of the observed universe has been a subject of fascination for physicists since the late 19th century. ${ }^{1}$ The following general time asymmetries are observed in this universe:

- The thermodynamic arrow of time - the fact that approximately isolated systems are now almost all evolving towards equilibrium in the same direction of time.

- The psychological arrow of time — we remember the past, we predict the future.

- The arrow of time of retarded electromagnetic radiation.

- The arrow of time supplied by the $C P$ non-invariance of the weak interactions and the $C P T$ invariance of field theory.

- The arrow of time of the approximately uniform expansion of the universe.

- The arrow of time supplied by the growth of inhomogeneity in the expanding universe.

All of these time asymmetries could arise from time-symmetric dynamical laws solved with time-asymmetric boundary conditions. The thermodynamic arrow of time, for example, is implied by an initial condition in which the progenitors of today's approximately isolated systems were all far from equilibrium at an initial time. The $C P$ arrow of time could arise as a spontaneously broken symmetry of the Hamiltonian. The approximate uniform expansion of the universe and the growth of inhomogeneity follow from an initial "big bang" of sufficient spatial homogeneity and isotropy, given the attractive nature of gravity. Characteristically such arrows of time can be reversed temporarily, locally, in isolated subsystems, although typically at an expense so great that the experiment can be carried out only in our imaginations. If we could, in the classical example of Loschmidt [4], reverse the momenta of all particles and fields of an isolated subsystem, it would "run backwards" with thermodynamic and electromagnetic arrows of time reversed.

Quantum cosmology is that part of physics concerned with the theory of the boundary conditions of our universe. It is, therefore, the natural and most general context in which to investigate the origin of observed time asymmetries. In the context of contemporary quantum cosmology, several such investigations have been carried out [2, 5, 6, 7, 8, 9, 10, 11], starting with those of Penrose [2] on classical time-asymmetric initial and final conditions and those of Page [5] and Hawking [6] on the emergence of the thermodynamic arrow of time from the "no-boundary" theory of the initial condition of the universe. It is not our purpose to review these results or the status of our understanding of the time asymmetries mentioned above. Rather, we shall discuss in this essay, from the perspective of quantum cosmology, a time asymmetry not specifically mentioned above. That is the arrow of time of familiar quantum mechanics.

Conventional formulations of quantum mechanics incorporate a fundamental distinction between the future and the past, as we shall review in Section II. This quantum-mechanical

\footnotetext{
${ }^{1}$ For clear reviews, see [1, 2, 3].
} 
arrow of time has, in a way, a distinct status in the theory from the time asymmetries discussed above. It does not arise, as they do, from a time-asymmetric choice of boundary conditions for time-neutral dynamical laws. Rather, it can be regarded as a time asymmetry of the laws themselves. However, the quantum mechanics of cosmology does not have to be formulated in this time-asymmetric way. In Section III, extending discussions of Aharonov, Bergman, and Lebovitz [12] and of Griffiths [13], we consider a generalized quantum mechanics for cosmology that utilizes both initial and final conditions to give a time-neutral, two-boundary formulation that does not necessarily have an arrow of time 14. In such a formulation all time asymmetries arise from properties of the initial and final conditions, in particular differences between them, or, at particular epochs, from nearness to the beginning or end. A theory of both initial and final conditions would be the objective of quantum cosmology.

In the context of a time-neutral formulation, the usual quantum mechanics results from utilizing a special initial condition, together with what amounts to a final condition representing complete indifference with respect to the future states, thus yielding the quantummechanical arrow of time, which is sufficient to explain the observed time asymmetries of this universe. However, a time-neutral formulation of quantum mechanics allows us to investigate to what extent the familiar final condition of indifference with respect to future states is mandated by our observations. In particular, it allows us to investigate whether quantum cosmologies with less blatantly asymmetric initial and final conditions might also be consistent with the observed general time asymmetries. As a step in this direction we discuss a quantum cosmology that would be, in a sense, the opposite extreme - a cosmology with a time-symmetric pair of initial and final conditions leading to a universe that is statistically symmetric about a moment of time. Such boundary conditions imply deviations from the thermodynamic arrow of time and the arrow of time supplied by the $C P$ non-invariance of the weak interactions. We investigate such deviations to see if they are inconsistent with observations. The classical statistical models reviewed in Section IV and the models of $C P$ symmetry breaking discussed in Section $\mathrm{V}$ suggest that the predicted deviations may be insufficient to exclude time-symmetric boundary conditions if the interval between initial and final conditions is much longer than our distance in time from the initial condition. Next, we review and augment the arguments of Davies and Twamley that electromagnetic radiation may supply a probe of the final condition that is sufficiently accurate to rule out time-symmetric boundary conditions.

We should emphasize that we are not advocating a time-symmetric cosmology but only using it as a foil to test the extent to which observation now requires the usual asymmetric boundary conditions and to search for more refined experimental tests. The important result of this paper is a quantum framework for examining cosmologies with less asymmetric boundary conditions than the usual ones, so that the quantum-mechanical arrow of time (with its consequent time asymmetries) can be treated, or derived, as one possibility out of many, to be confronted with observation, rather than as an axiom of theory.

Relations between the initial and final conditions of a quantum-mechanical universe sufficient for both CPT-symmetric cosmologies and time-symmetric cosmologies are discussed in Section V. Ways in which the $T$-violation exhibited by the weak interaction could arise in such universes are described there as well. In Section VI we discuss the limitations on time-symmetric quantum boundary conditions following from the requirements of decoherence and classicality. Specifically, we show that for a set of alternative histories to have 
the negligible interference between its individual members that is necessary for them to be assigned probabilities at all, there must be some impurity in the initial or final density matrices or both, except in the highly unorthodox case in which there are only one or two coarse-grained histories with non-negligible probability.

We should make clear that our discussion of time-symmetric cosmologies, based on speculative generalizations of quantum mechanics and causality, with separate initial and final density matrices that are related by time symmetry, is essentially different from the conjecture that has sometimes been made that ordinary causal quantum or classical mechanics, with just a single boundary condition or a single prescribed wave function, $C P T$-invariant about some time in the distant future, might lead to a $T$-symmetric or $C P T$-symmetric cosmology with a contracting phase in which the arrows of time are reversed [6, 15, 16, 17, 18]. It is the latter notion, by the way, that Hawking refers to as his "greatest mistake" [19]. We shall return to this topic in Section V.

\section{THE ARROW OF TIME IN QUANTUM MECHANICS}

As usually formulated, the laws of quantum mechanics are not time-neutral but incorporate an arrow of time. This can be seen clearly from the expression for the probabilities of histories consisting of alternatives at definite moments of time $t_{1}<t_{2}<\cdots<t_{n}$. Let $\left\{\alpha_{k}\right\}$ be an exhaustive set of alternatives at time $t_{k}$ represented by $\left\{P_{\alpha_{k}}^{k}\left(t_{k}\right)\right\}$, a set of projection operators in the Heisenberg picture. For example, the alternatives $\left\{\alpha_{k}\right\}$ might be defined by an exhaustive set of ranges for the center-of-mass position of a massive body. A particular history corresponds to a specific sequence of alternatives $\left(\alpha_{1}, \cdots, \alpha_{n}\right)$. The probability for a particular history in the exhaustive set of histories is

$$
p\left(\alpha_{n}, \cdots, \alpha_{1}\right)=\operatorname{Tr}\left[P_{\alpha_{n}}^{n}\left(t_{n}\right) \cdots P_{\alpha_{1}}^{1}\left(t_{1}\right) \rho P_{\alpha_{1}}^{1}\left(t_{1}\right) \cdots P_{\alpha_{n}}^{n}\left(t_{n}\right)\right],
$$

where $\rho$ is the density matrix describing the initial state of the system and the projection operators are time-ordered from the density matrix to the trace. ${ }^{2}$

The expression for the probabilities (2.1) is not time-neutral. This is not because of the time ordering of the projection operators. Field theory is invariant under $C P T$ and the ordering of the operators could be reversed by a CPT transformation of the projection operators and density matrix, leaving the probabilities unchanged. (See e.g. [20] or [14]). Either time ordering may therefore be used; it is by convention that we usually use the one with the condition represented by the density matrix $\rho$ in the past.

Rather, (2.1) is not time-neutral because there is a density matrix on one end of the chain of projections representing a history while at the other end there is the trace [12, 13, 14]. Whatever conventions are used for time ordering, there is thus an asymmetry between future and past exhibited in the formula for probabilities (2.1). That asymmetry is the arrow of time in quantum mechanics.

\footnotetext{
${ }^{2}$ This compact expression of the probabilities of ordinary quantum mechanics has been noted by many authors. For more details of this and other aspects of the quantum mechanical formalism we shall employ the reader is referred to [20] and [14] where references to earlier literature may be found.
} 
The asymmetry between past and future exhibited by quantum mechanics implies the familiar notion of causality. From an effective density matrix describing the present alone it is possible to predict the probabilities for the future. More precisely, given that alternatives $\alpha_{1}, \cdots, \alpha_{k}$ have "happened" at times $t_{1}<\cdots<t_{k}$ before time $t$, the conditional probability for alternatives $\alpha_{k+1}, \cdots, \alpha_{n}$ to occur in the future at times $t_{k+1}, \cdots, t_{n}$ may be determined from an effective density matrix $\rho_{\text {eff }}(t)$ at time $t$. Specifically, the conditional probabilities for future prediction are

$$
p\left(\alpha_{n}, \cdots, \alpha_{k+1} \mid \alpha_{k}, \cdots, \alpha_{1}\right)=\frac{p\left(\alpha_{n}, \cdots, \alpha_{1}\right)}{p\left(\alpha_{k}, \cdots, \alpha_{1}\right)} .
$$

These can be expressed as

$$
p\left(\alpha_{n}, \cdots, \alpha_{k+1} \mid \alpha_{k}, \cdots, \alpha_{1}\right)=\operatorname{Tr}\left[P_{\alpha_{n}}^{n}\left(t_{n}\right) \cdots P_{\alpha_{k+1}}^{k+1}\left(t_{k+1}\right) \rho_{\mathrm{eff}}\left(t_{k}\right) P_{\alpha_{k+1}}^{k+1}\left(t_{1}\right) \cdots P_{\alpha_{n}}^{n}\left(t_{n}\right)\right]
$$

where the effective density matrix $\rho_{\text {eff }}$ is

$$
\rho_{\text {eff }}\left(t_{k}\right)=\frac{P_{\alpha_{k}}^{k}\left(t_{k}\right) \cdots P_{\alpha_{1}}^{1}\left(t_{1}\right) \rho P_{\alpha_{1}}^{1}\left(t_{1}\right) \cdots P_{\alpha_{k}}^{k}\left(t_{k}\right)}{\operatorname{Tr}\left[P_{\alpha_{k}}^{k}\left(t_{k}\right) \cdots P_{\alpha_{1}}^{1}\left(t_{1}\right) \rho P_{\alpha_{1}}^{1}\left(t_{1}\right) \cdots P_{\alpha_{k}}^{k}\left(t_{k}\right)\right]} .
$$

The density matrix $\rho_{\text {eff }}\left(t_{k}\right)$ can be said to define the effective state of the universe at time $t_{k}$, given the history $\left(\alpha_{1}, \cdots, \alpha_{k}\right)$.

What is the physical origin of the time asymmetry in the basic laws of quantum mechanics and what is its connection with the other observed time asymmetries of our universe? The rest of this Section addresses that question.

The reader may be most familiar with the expression for probabilities (2.1) in the context of the approximate "Copenhagen" quantum mechanics of measured subsystems. In that case operators, the density matrix, etc. all refer to the Hilbert space of the subsystem. The sets of projection operators $\left\{P_{\alpha_{k}}^{k}\left(t_{k}\right)\right\}$ describe alternative outcomes of measurements of the subsystem.

Formula (2.1) for the probabilities of a sequence of measured outcomes is then a unified expression of the "two forms of evolution" usually discussed in the quantum mechanics of subsystems - unitary evolution in between measurements and the "reduction of the state vector" on measurement. The time asymmetry of (2.1) does not arise from the unitary evolution of the projection operators representing the measured quantities in the Heisenberg picture; that is time-reversible. Rather, it can be said to arise from the successive reductions represented by the projections in (2.4) that occur on measurement. The common explanation for the origin of the arrow of time in the quantum mechanics of measured subsystems is that measurement is an irreversible process and that quantum mechanics inherits its arrow of time from the arrow of time of thermodynamics. ${ }^{3}$ If that is the case, then the origin of the

3 This connection between the thermodynamic arrow of time and the quantum-mechanical arrow of time can be ambiguous. Suppose, for example, a measuring apparatus is constructed in which the local approach to equilibrium is in the opposite direction of time from that generally prevailing in the larger universe. If that apparatus interacts with a subsystem (perhaps previously measured by other apparatus adhering to the general thermodynamic arrow of time) should the operators representing those measurements be 
quantum-mechanical arrow of time must ultimately be cosmological, for the straightforward explanation of the thermodynamic arrow of time is a special initial condition for the universe implying that its constituents were far from equilibrium across a spacelike surface. Let us, therefore, investigate more fundamentally the quantum-mechanical arrow of time, not in an approximate quantum mechanics of measured subsystems, but in the quantum mechanics of a closed system - most realistically and generally the universe as a whole.

The formula (2.1) for the probabilities of histories also holds in the quantum mechanics of a closed system such as the universe as a whole, at least in an approximation in which gross fluctuations in the geometry of spacetime are neglected. The sets of projection operators describe alternatives for the whole system, say the universe, and the density matrix can be thought of as describing its initial condition. ${ }^{4}$ Not every set of histories that may be described can be assigned probabilities according to eqreftwoone. In the quantum mechanics of closed systems consistent probabilities given by (2.1) are predicted only for those sets of histories for which there is negligible interference between the individual members of the set [14] as a consequence of the particular initial $\rho$. Such sets of histories are said to "decohere". We shall defer until Section $\mathrm{V}$ a discussion of the precise measure of the coherence between histories and the implications of decoherence for time symmetry in quantum mechanics. We concentrate now on the theoretical status of the arrow of time exhibited by (2.1) in the quantum mechanics of cosmology.

An arrow of time built into a basic quantum mechanics of cosmology may not (as in the approximate "Copenhagen" quantum mechanics of measured subsystems) be attributed to the thermodynamic arrow of an external measuring apparatus or larger universe. In general, these external objects are not there. An arrow of time in the quantum mechanics of cosmology would be a fundamental time asymmetry in the basic laws of physics. Indeed, given that, as we mentioned in the Introduction, the other observed time asymmetries could all arise from time-symmetric dynamical laws solved with time-asymmetric boundary conditions, a fundamental arrow of time in the laws of quantum mechanics could be the only fundamental source of time asymmetry in all of physics.

There is no inconsistency between known data and a fundamental arrow of time in quantum mechanics. General time asymmetries are exhibited by our universe and there is no evidence suggesting any violation of causality. The observed time asymmetries such as the thermodynamic arrow of time, the arrow of retarded electromagnetic radiation, the absence of white holes, etc. could all be seen to follow from a fundamental quantum-mechanical distinction between the past and future. That is, they could all be seen to arise from a special initial $\rho$ in a quantum-mechanical framework based on (2.1).

But might it not be instructive to generalize quantum mechanics so that it does not so blatantly distinguish past from future? One could then investigate a more general class of quantum cosmologies and identify those that are compatible with the observed time asymmetries. Even if it is highly unlikely that ordinary quantum mechanics needs to be

ordered according to the thermodynamic arrow of the apparatus or of the larger universe with respect to which it is running backwards? Such puzzles are resolvable in the more general quantum mechanics of closed systems to be discussed below, where "measurements", the "thermodynamic arrow of time", and any connection between the two are all approximate notions holding in only special situations.

${ }^{4}$ For a more detailed exposition of this quantum mechanics of cosmology, the reader is referred to our previous work 14, 20, 21], where references to the earlier literature may also be found. 
replaced by such a generalization, the generalization can still provide an instructive way of viewing the origin of time asymmetry in the universe and provide a framework for discussing tests of the usual assumptions. We shall discuss in the next section a quantum mechanics that employs two boundary conditions, one for the past and one for the future, to give a time-neutral formulation. Each condition is represented by a density matrix and the usual theory is recovered when the future density matrix is proportional to the unit matrix while the one for the past is much more special.

\section{A TIME-NEUTRAL FORMULATION OF QUANTUM MECHANICS FOR COSMOLOGY}

Nearly thirty years ago, Aharonov, Bergmann, and Lebovitz [12] showed how to cast the quantum mechanics of measured subsystems into time-neutral form by considering final conditions as well as initial ones. The same type of framework for the quantum mechanics of closed systems has been discussed by Griffiths [13] and ourselves [14]. In this formulation the probabilities for the individual members of a set of alternative histories is given by

$$
p\left(\alpha_{n}, \cdots, \alpha_{1}\right)=N \operatorname{Tr}\left[\rho_{f} P_{\alpha_{n}}^{n}\left(t_{n}\right) \cdots P_{\alpha_{1}}^{1}\left(t_{1}\right) \rho_{i} P_{\alpha_{1}}^{1}\left(t_{1}\right) \cdots P_{\alpha_{n}}^{n}\left(t_{n}\right)\right]
$$

where

$$
N^{-1}=\operatorname{Tr}\left(\rho_{f} \rho_{i}\right)
$$

Here, $\rho_{i}$ and $\rho_{f}$ are Hermitian, positive operators that we may conventionally call Heisenberg operators representing initial and final conditions for the universe respectively. They need not be normalized as density matrices with $\operatorname{Tr}(\rho)=1$ because (3.1) is invariant under changes of normalization.

The expression (3.1) for the probabilities of histories is time-neutral. There is a density matrix at both ends of each history. Initial and final conditions may be interchanged by making use of the cyclic property of the trace. Therefore, the quantum mechanics of closed systems based on (3.1) need not have a fundamental arrow of time.

Different quantum-mechanical theories of cosmology are specified by different choices for the initial and final conditions $\rho_{i}$ and $\rho_{f}$. For those cases with $\rho_{f} \propto I$, where $I$ is the unit matrix, this formulation reduces to that discussed in the previous Section because then (3.1) coincides with (2.1).

Of course, the condition for decoherence must also be extended to incorporate initial and final conditions. That extension, however, is straightforward [13, 14] and will be reviewed briefly in Section V. The result is a generalized quantum mechanics in the sense of Refs. 14] and [21].

Lost in this generalization is a built-in notion of causality in quantum mechanics. Lost also, when $\rho_{f}$ is not proportional to $I$, is any notion of a unitarily evolving "state of the system at a moment of time". There is generally no effective density matrix like $\rho_{\text {eff }}(t)$ in (2.4) from which alone probabilities for either the future or past could be computed. What is gained is a quantum mechanics without a fundamental arrow of time in which all time asymmetries may arise in particular cosmologies because of differences between $\rho_{i}$ and $\rho_{f}$ or at particular epochs from their being near to the beginning or the end. That 
generalized quantum mechanics embraces a richer variety of possible universes, allowing for the possibility of violations of causality and advanced as well as retarded effects. These, therefore, become testable features of the universe rather than axioms of the fundamental quantum framework.

From the perspective of this generalized quantum mechanics the task of quantum cosmology is to find a theory of both the initial and final conditions that is theoretically compelling and fits our existing data as well as possible. Certainly a final condition of indifference, $\rho_{f} \propto I$, and a special initial condition, $\rho_{i}$, seem to fit our data well, and there is no known reason for modifying them. But how accurately is $\rho_{f} \propto I$ mandated by the data? What would be the observable consequences of a completely time-symmetric boundary condition that is, in a sense, the opposite extreme?

Our ability to detect the presence of a final condition differing from $\rho_{f} \propto I$ depends on our experimental access to systems whose behavior today predicted with $\rho_{f} \not \subset I$ would be measurably different from the predictions of that behavior with $\rho_{f} \propto I$. Loosely speaking, it depends on our finding physical systems which can "see" the final condition of the universe today. In the following we examine several candidates for such systems, beginning with simple classical analyses in Section IV and proceeding to more quantum-mechanical ones in Section V.

\section{CLASSICAL TWO-TIME BOUNDARY PROBLEMS}

\section{A. A Simple Statistical Model}

The simplest explanation of the observed thermodynamic arrow of time is the asymmetry between a special, low-entropy, ${ }^{5}$ initial condition and a maximal-entropy final condition describable as indifference with respect to final state (or as no condition at all!). Studying deviations of the entropy increase predicted by statistical mechanics with these boundary conditions from that predicted with time-symmetric boundary conditions is a natural way to try to discriminate between the two. Such studies were carried out in classical statistical models by by Cocke [22], Schulman [23], Wheeler [24], and others in the late '60s and early '70s. Schulman, in particular, has written extensively on these problems both in classical and quantum mechanics [25]. We briefly review such statistical models here.

Relaxation to equilibrium is a time-symmetric process in a universe with an underlying dynamics that is time reversal invariant. Without boundary conditions, a system out of equilibrium is just as likely to have evolved from a state of higher entropy as it is to evolve to a state of higher entropy. The characteristic relaxation time for a system to evolve to equilibrium depends on the size of the system and the strength of the interactions that equilibrate it. Other factors being equal, the larger the system the longer the relaxation time.

There is no simpler instructive model to illustrate the approach to equilibrium than the Ehrenfest urn model [26]. For this reason, it and related models have been much studied in connection with statistical two-time boundary problems [22, 23]. The model consists of

\footnotetext{
${ }^{5}$ For quantitative estimates of how low the initial entropy is, see [2].
} 
two boxes and a numbered set of $n$ balls that are distributed between them. The system evolves in time according to the following dynamical rule: At each time step a random number between 1 and $n$ is produced, and the ball with that number is moved from the box containing it to the other box. This dynamical rule is time-symmetric.

The fine-grained description of this system specifies which ball is in which box (a "microstate"). An interesting coarse-grained description involves following just the total number of balls in each box ( a "macrostate") irrespective of which balls are in which box. Let us use this coarse graining to consider an initial condition in which all the balls are in one box and follow the approach to equilibrium as a function of the number of time steps, with no further conditions. Figure 1 shows a numerical calculation of how the entropy averaged over many realizations of this evolution grows with time to approach its maximum, equilibrium value. The relaxation time, obtained either analytically or numerically, is approximately the total number of balls, $t_{\text {relax }} \sim n$. If there are no further constraints, the system tends to relax to equilibrium and remain there.

Consider evolution in the Ehrenfest urn model when a final condition identical to the initial one is imposed at a later time $T$. Specifically, construct an ensemble of evolutions consistent with these boundary conditions by evolving forward from an initial condition where all the balls are in one box but accepting only those evolutions where all the balls are back in this box at time $T$. Figure 1 shows the results of two such calculations, one for a system with a small number of balls (where the relaxation time is significantly smaller than $T$ ) and the other for a system with a larger number of balls (where it is significantly larger than $T$.)

For both systems the time-symmetric boundary conditions imply a behavior of the average entropy that is time-symmetric about the midpoint, $T / 2$. For the system with a relaxation time short compared to the time at which the final condition is imposed, the initial approach to equilibrium is nearly indistinguishable from that in the case with no final condition. That is because, in equilibrium, the system's coarse-grained dynamics is essentially independent of its initial or final condition. It, in effect, "forgets" both from whence it started and whither it is going.

By contrast, if the relaxation time is comparable to or greater than the time interval between initial and final condition, then there will be significant deviations from the unconstrained approach to equilibrium. Such systems typically do not reach equilibrium before the effect of the final condition forces their entropy to decrease.

The evolution of the entropy in the presence of time-symmetric initial and final conditions must itself be time-symmetric when averaged over many evolutions, as the simulations in Figure 1 show. However, in a statistical theory with time-symmetric boundary conditions the individual histories need not be time-symmetric. Figure 2 shows an example of a single history from an urn model calculation for which the average behavior of the entropy is shown in Figure 1. The ensemble of histories is time-symmetric by construction; the individual histories need not be. Since, by definition, we experience only one history of the universe, this leaves open the possibility that the time-asymmetries that we see could be the result of a statistical fluctuation in a universe with time-symmetric boundary conditions. In quantum cosmology, we would not count such a theory of the initial and final conditions as successful if the fluctuations required were very improbable. However, in some examples the magnitude of the fluctuation need not be so very large. For instance, consider a classical statistical theory in which the boundary conditions allow an ensemble of classical histories each one of which 

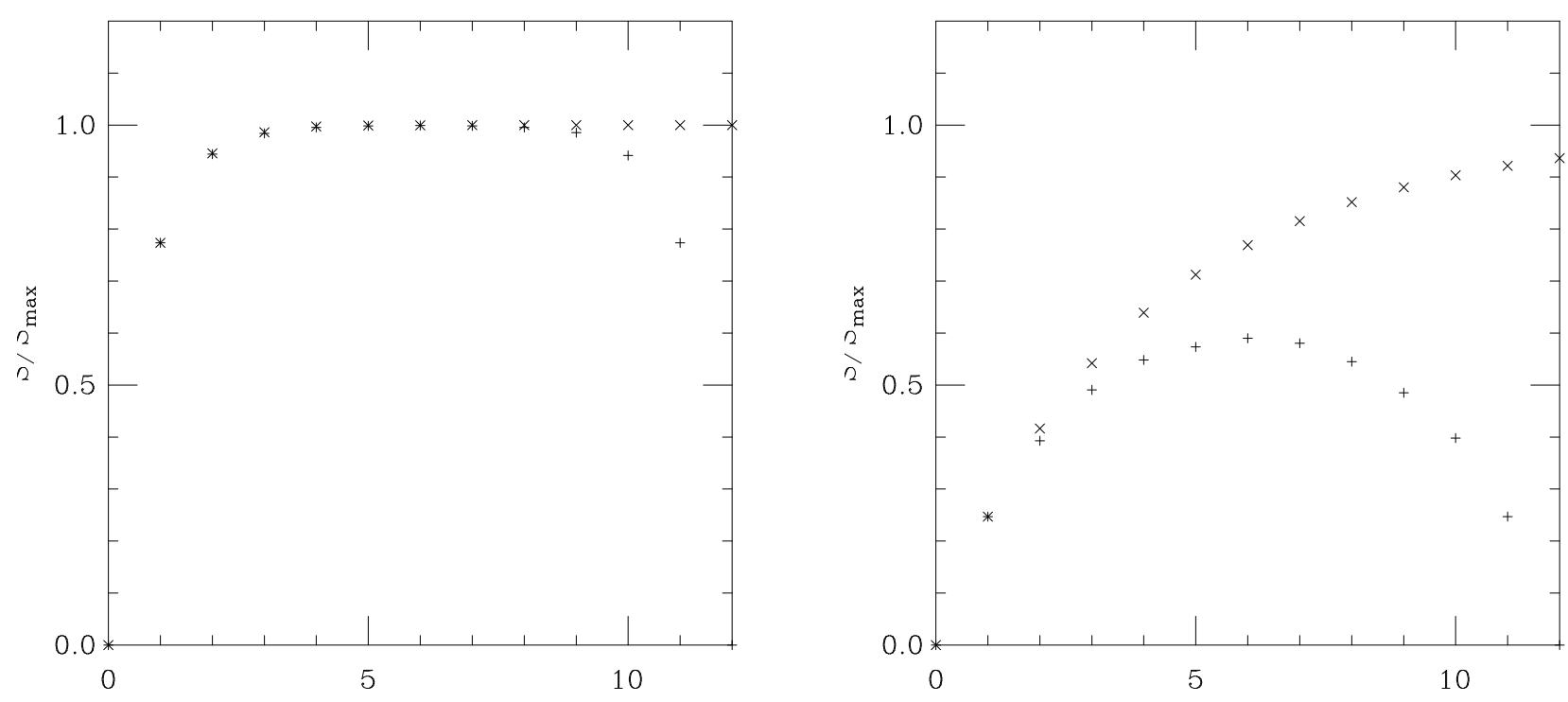

Figure 1: Simulation of the approach to equilibrium in the Ehrenfest urn model. The entropy of a coarse-grained state in which only the total number of balls in each box is followed is the logarithm number of different ways of distributing the balls between the boxes consistent with a given total number in each. This figure shows this entropy averaged over a large number of different evolutions of the system for several situations. These simulations were carried out by the authors but are no different in spirit from those discussed by Cocke [22].

The left figure shows the evolution of a system of four balls. In each case the system starts with all balls in one box - a configuration of zero entropy as far from equilibrium as it is possible to get. The $\times$ 's show how the average entropy of 12,556 cases approaches equilibrium when there are no further constraints. The entropy approaches its equilibrium value in a relaxation time given approximately by its size, $t_{\text {relax }} \sim 4$, and remains there. The curve of + 's shows the evolution when a time symmetric final condition is imposed at $T=12$ that all balls have returned to the one box from whence they started at $t=0$. A total of 100,000 evolutions were tried.

The average entropy of the 12,556 cases that met the final condition is shown. It is time symmetric about the midpoint, $T / 2=6$. The initial approach to equilibrium is virtually indistinguishable from the approach without a final condition because $t_{\text {relax }} \sim 4$ is significantly less than the time $T=12$ at which the final condition is imposed. Only within one relaxation time of the final condition is the deviation of the evolution from the unconstrained case apparent.

The right figure shows the same two cases for a larger system of twenty balls. The unconstrained approach to equilibrium shown by the $X$ 's was calculated from the average of 1010 evolutions and exhibits a relaxation time, $t_{\text {relax }} \sim 20$. The average entropy when time-symmetric boundary conditions are imposed at $T=12$ is shown in the curve of +'s. 10,000,000 evolutions were tried of which 1010 met the time-symmetric final condition. (This demonstrates vividly that it is very improbable for the entropy of even a modest size system to fluctuate far from equilibrium as measured by the $C P U$ time needed to find such fluctuations.)

The deviation from the unconstrained approach to equilibrium caused by the imposition of a timesymmetric final condition is significant from an early time of about $t=3$ as the differences between the +'s and the $\times$ 's show. These models suggest that to detect the effects of a time symmetric final condition for the universe we must have access to systems for which the relaxation time is at least comparable to the time difference between initial and final conditions.

displays an arrow of time and also, with equal probability, the time-reversed of that history displaying the opposite arrow of time. The boundary conditions and the resulting ensemble are time-symmetric, but predict an observed time-asymmetry with probability one. Of course, such a theory might be indistinguishable from one that posited boundary conditions 
allowing just one arrow of time. However, other theoretical considerations may make it reasonable to consider such proposals, for example, the "no boundary" initial condition which is believed to have this character [5]. In the subsequent discussion of time-symmetric cosmological boundary conditions we shall assume that they predict with high probability some observable differences from the usual special initial conditions and final condition of indifference and investigate what these are.

\section{B. Classical Dynamical Systems with Two-Time Statistical Boundary Conditions}

The analysis of such simple classical statistical models with two-time boundary conditions suggests the behavior of a classical cosmology with time-symmetric initial and final conditions. A classical dynamical system is described by a phase space and a Hamiltonian, $H$. We write $z=(q, p)$ for a set of canonical phase-space coördinates. The histories of the classical dynamical system are the phase-space curves $z(t)$ that satisfy Hamilton's equations of motion.

A statistical classical dynamical system is described by a distribution function on phase space $\rho^{c l}(z)$ that gives the probability of finding it in a phase-space volume. For analogy with quantum mechanics it is simplest to use a "Heisenberg picture" description in which the distribution function does not depend explicitly on time but time-dependent coördinates on phase space are used to describe the dynamics. That is, if $z_{0}$ is a set of canonical coördinates at time $t=0$, a set $z_{t}$ appropriate to time $t$ may be defined by $z_{t}=z_{0}(t)$ where $z_{0}(t)$ is the classical evolution of $z_{0}$ generated in a time $t$ by the Hamiltonian $H$. The statistical system at time $t$ is then distributed according to the function $\rho^{c l}$ expressed in terms of the coördinates $z_{t}$, viz. $\rho^{c l}\left(z_{t}\right)$. The distributions $\rho^{c l}\left(z_{t}\right)$ and $\rho^{c l}\left(z_{t^{\prime}}\right)$ will therefore typically have different functional forms.

An ensemble of histories distributed according to the probabilities of a statistical classical dynamical system with boundary conditions at two times $t_{i}$ and $t_{f}$ might be constructed as follows: Evolve a large number of systems distributed according to the initial distribution function $\rho_{i}^{c l}\left(z_{i}\right)$ forward from time $t_{i}$ to time $t_{f}$. If a particular system arrives at time $t_{f}$ in the phase space volume $\Delta v$ centered about point $z_{f}$, select it for inclusion in the ensemble with probability $\rho_{f}^{c l}\left(z_{f}\right) \Delta v$ where $\rho_{f}^{c l}$ is the distribution function representing the final boundary condition. Thus, if $\rho_{i}^{c l}$ and $\rho_{f}^{c l}$ are referred to a common set of phase-space coördinates, say $z_{t}$, the time-symmetric ensemble of systems will be distributed according to the function

$$
\bar{\rho}^{c l}\left(z_{t}\right)=N \rho_{f}^{c l}\left(z_{t}\right) \rho_{i}^{c l}\left(z_{t}\right),
$$

where

$$
N^{-1}=\int d z_{t} \rho_{f}^{c l}\left(z_{t}\right) \rho_{i}^{c l}\left(z_{t}\right) .
$$

Referred to the initial time, (4.1) has a simple interpretation: Since classical evolution is unique and deterministic the selection at the final time could equally well be carried out at the initial time with $\rho_{f}^{c l}$ evolved back to the initial time. The distribution $\bar{\rho}^{c l}$ is the result.

We now discuss the relation between $\rho_{i}^{c l}$ and $\rho_{f}^{c l}$ that is necessary for the probabilities of this classical cosmology to be symmetric about a moment of time. Take this time to be $t=0$ and introduce the operation, $\mathcal{T}$, of time-reversal about it.

$$
\mathcal{T} \rho^{c l}\left(q_{0}, p_{0}\right) \equiv \rho^{c l}\left(q_{0},-p_{0}\right) .
$$


If we assume that the Hamiltonian is itself time-reversal invariant

$$
H\left(q_{0}, p_{0}, t\right)=H\left(q_{0},-p_{0},-t\right),
$$

this implies

$$
\mathcal{T} \rho^{c l}\left(q_{t}, p_{t}\right)=\rho^{c l}\left(q_{-t},-p_{-t}\right) .
$$

The distribution function (4.1) may then be conveniently rewritten

$$
\bar{\rho}^{c l}\left(q_{t}, p_{t}\right)=N \rho_{i}^{c l}\left(q_{t}, p_{t}\right) \mathcal{T} \rho_{f}^{c l}\left(q_{-t},-p_{-t}\right) .
$$

A relation between $\rho_{i}^{c l}$ and $\rho_{f}^{c l}$ sufficient to imply the time-symmetry of the distribution $\bar{\rho}^{c l}$ is now evident, namely

$$
\rho_{f}^{c l}\left(q_{t}, p_{t}\right)=\mathcal{T}^{-1} \rho_{i}^{c l}\left(q_{t}, p_{t}\right) .
$$

The final condition is just the time-reversed version of the initial one.

The imposition of time-symmetric statistical boundary conditions on a classical cosmology means in particular that the entropy must behave time-symmetrically if it is computed utilizing a coarse graining that is itself time-symmetric. The entropy of the final distribution must be the same as the initial one. The thermodynamic arrow of time will run backwards on one side of the moment of time symmetry as compared to the other side. This does not mean, of course, that the histories of the ensemble need be individually time-symmetric, as the example in Figure 2 shows. In particular, subsystems with relaxation times long compared to the interval between initial and final conditions might have non-negligible probabilities for fluctuations from exactly time-symmetric behavior. There would appear to be no principle, for example, forbidding us to live on into the recontracting phase of the universe and see it as recontracting. It is just that as time progressed events judged to be unexpected on the basis of just an initial condition would happen with increasing frequency. It is by the frequency of such unexpected events that we could detect the existence of a final condition.

Could we infer the existence of a time-symmetric final condition for the universe from the deviations that it would imply for the approach to equilibrium that would be expected were there no such final condition? The statistical models reviewed in above suggest that to do so we would need to study systems with relaxation times comparable to or longer than the lifetime of the universe between the "big bang" and the "big crunch". If the lifetime of the universe is comparable to the present age of the universe from the "big bang", then we certainly know such systems. Systems of stars such as galaxies and clusters provide ready examples. Any single star, with the ambient radiation, provides another as long as the star's temperature is above that of the cosmic background radiation. Black holes with lifetime to decay by the Hawking radiation longer than the Hubble time are further examples. Indeed, from the point of view of global cosmological geometry, the singularities contained within black holes can be considered to be parts of the final singularity of the universe, where a final condition would naturally be imposed [27]. The singularities of detectable black holes may be the parts of this final singularity closest to us. On smaller scales, samples of radioactive material with very long half-lives may be other such examples and Wheeler 24] has discussed experiments utilizing them to search for a time-symmetric final condition. We may hope, as mentioned above, that the evolving collective complex adaptive system of which we are a part could be such a long-lasting phenomenon! 


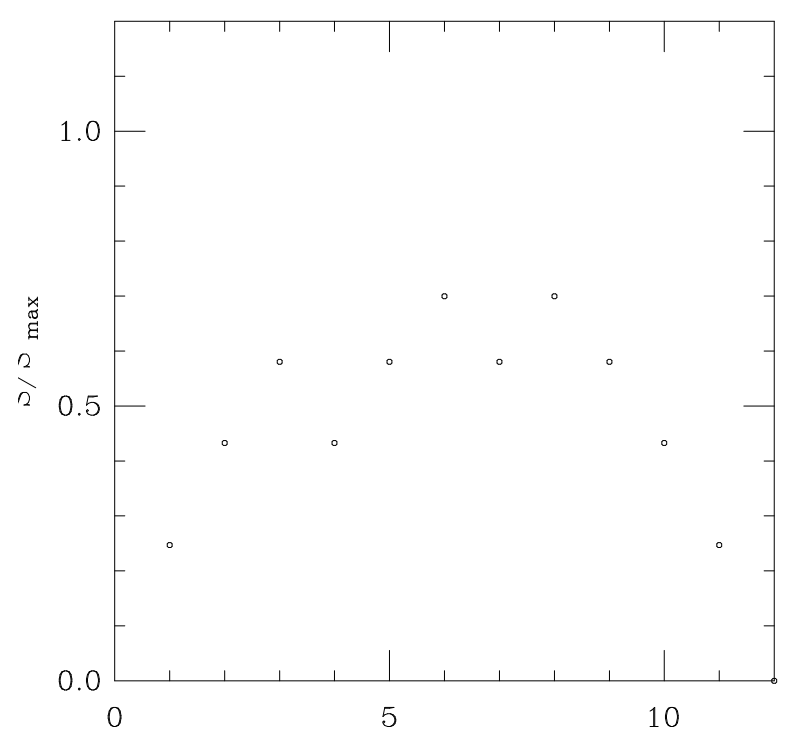

Fig 2. An individual evolution in the urn model with time-symmetric initial and final conditions. The history of the entropy averaged over many evolutions must clearly be time-symmetric in a statistical theory with time-symmetric initial and final conditions as Figure 1 shows. However, the individual evolutions need not be separately time-symmetric. This figure shows the entropy for the case of twenty balls in the first evolution among the 10,000,000 described in Figure 1 that met the time-symmetric final condition. It is not time-symmetric. For systems such as this with relaxation time, $t_{\text {relax }}$, larger than the time between initial and final conditions, significant deviations from exact time symmetry may be expected.

However, if the lifetime of the universe is much longer than the present age from the "big bang", then it might be much more difficult to find systems that remain out of equilibrium long enough for their initial approach to equilibrium to be significantly affected by a timesymmetric final condition. That could be the case with the $\Omega$-near-one universe that would result from a rapid initial inflation. If its lifetime were long enough, we might never be able to detect the existence of a time-symmetric final condition for the universe.

The lifetime of our classical universe obeying the Einstein equation is, of course, in principle determinable from present observations (for example, of the Hubble constant, mean mass density, and deceleration parameter). Unfortunately we do not have enough data to distinguish observationally between a lifetime of about twenty-five billion years and an infinite lifetime. Very long lifetimes are not only consistent with observations, but also, as we now describe, are suggested theoretically by quantum cosmology as a consequence of inflation.

The quasiclassical cosmological evolution that we observe should be, on a more fundamental level, a likely prediction of a quantum theory of the universe and its boundary conditions. We shall discuss time symmetry in the context of quantum cosmology in later sections, but for the present discussion we note that, in quantum cosmology, the probabilities for different lifetimes of the universe are predictable from a theory of its initial and final conditions. That is because in a quantum theory that includes gravitation the geometry of spacetime, including such features as the time between the "big bang" and a "big crunch", if any, is quantum-probabilistic. 
A quantum state that predicts quasiclassical behavior does not typically predict a unique classical history but rather an ensemble of possible classical histories with different probabilities. This is familiar from wave functions of WKB form, which do not predict single classical trajectories but only the classical connection between momentum and classical action. Similarly, in the quantum mechanics of closed cosmologies, we expect a theory of quantum boundary conditions to determine an ensemble of different classical cosmological geometries with different probabilities. ${ }^{6}$ The geometries in the ensemble will have different times between the "big bang" and the "big crunch" because in quantum gravity that time is a dynamical variable and not a matter of our choice. In this way, the probability distribution of lifetimes of the universe becomes predictable in quantum cosmology.

Cosmological theories that predict inflation lead to very large expected values for the lifetime of the universe; and inflation seems to be implied by some currently interesting theories of the boundary conditions of the universe. The question has been analyzed only for theories with a special initial condition, such as the "no-boundary proposal" and the "tunneling-from-nothing proposal". Analyses by Hawking and Page [10], Grishchuk and Rozhansky [28], and Barvinsky and Kamenshchik [29] suggest very large expected lifetimes for the "no-boundary proposal". Analyses by Vilenkin [30] and by Mijić, Morris, and Suen 31] do likewise for the "tunneling-from-nothing" case.

\section{Electromagnetic Radiation}

The above discussion suggests that in order to probe the nature of a non-trivial final condition, one should study processes today that are sensitive to that final condition no matter how far in the future it is imposed. At the conference, P.C.W. Davies suggested that electromagnetic radiation might provide such a mechanism for "seeing" a final condition in the arbitrarily far future in realistic cosmologies. In an approximately static and homogeneous cosmology, radiation must travel through ever more material the longer the time separation between initial and final conditions. For sufficiently large separations, the universe becomes opaque to the electromagnetic radiation necessary to probe the details of the final condition directly. However, in an expanding universe the dilution of matter caused by the expansion competes with the longer path length as the separation between initial big bang and final big crunch becomes longer and longer. Davies and Twamley [32] show that, under reasonable conditions, the expansion wins and that the future light cone is transparent to photons all the way to a distance from the final singularity comparable to ours from the big bang.

Transparency of the forward light cone raises the possibility of constraining the final condition by present observations of electromagnetic radiation and perhaps ruling out timesymmetric boundary conditions. Partridge [33] has actually carried out experiments which could be interpreted in this way and Davies and Twamley discuss others. The following is an example of a further argument of a very direct kind.

Suppose the universe to have initial and final classical distributions that are timesymmetric in the sense of (4.6). Suppose further that these boundary conditions imply with high probability an initial epoch with stars in galaxies distributed approximately ho-

\footnotetext{
${ }^{6}$ For further discussion see e.g. [14].
} 
mogeneously and a similar final epoch of stars in galaxies at the symmetric time. Consider the radiation emitted from a particular star in the present epoch. If the universe is transparent, it is likely to reach the final epoch without being absorbed or scattered. There it may either be absorbed in the stars or proceed past them towards the final singularity. If a significant fraction of the radiation proceeds past, then by time-symmetry we should expect a corresponding amount of radiation to have been emitted from the big bang. Observations of the brightness of the night sky could therefore constrain the possibility of a final boundary condition time-symmetrically related to the initial one. The alternative that the radiation is completely absorbed in future stars implies constraints on present emission that are probably inconsistent with observation because the total cross section of future stars is only a small fraction of the whole sky, as it is today. ${ }^{7}$

By such arguments, made quantitative, and extended to neutrinos, gravitational and other forms of radiation, we may hope to constrain the final condition of the universe no matter how long the separation between the big bang and the big crunch.

\section{HYPOTHETICAL QUANTUM COSMOLOGIES WITH TIME SYMMETRIES}

\section{A. $C P T$ - and T-Symmetric Boundary Conditions}

The time-neutral generalized quantum mechanics with initial and final conditions developed in Section III permits the construction of model quantum cosmologies that exhibit symmetries with respect to reflection about a moment of time. By this we mean that the probabilities given by (3.1) for a set of alternative histories are identical to those of the symmetrically related set. This section explores the relations between $\rho_{f}$ and $\rho_{i}$ and the conditions on the Hamiltonian under which such symmetries exist.

$C P T$-symmetric universes are the most straightforward to implement because local field theory in flat spacetime is invariant under $C P T$. We expect $C P T$ invariance as well for field theories in curved cosmological spacetimes such as closed Friedmann universes that are symmetric under a space inversion and symmetric about a moment of time.

To construct a CPT-invariant quantum cosmology, choose the origin of time so that the time reflection symmetry is about $t=0$. Let $\Theta$ denote the antiunitary $C P T$ transfor-

mation and for simplicity consider alternatives $\left\{P_{\alpha_{k}}^{k}\left(t_{k}\right)\right\}$ such that their CPT transforms, $\left\{\widetilde{P}_{\alpha_{k}}^{k}\left(-t_{k}\right)\right\}$, are given by

$$
\widetilde{P}_{\alpha_{k}}^{k}\left(-t_{k}\right)=\Theta^{-1} P_{\alpha_{k}}^{k}\left(t_{k}\right) \Theta .
$$

A CPT-symmetric universe would be one in which the probabilities of histories of alternatives at times $t_{1}<t_{2}<\cdots<t_{n}$ would be identical to the probabilities of the $C P T$ transformed histories of alternatives at times $-t_{n}<\cdots<-t_{2}<-t_{1}$. Denote by $C_{\alpha}$ the string of projection operators representing one history

$$
C_{\alpha}=P_{\alpha_{n}}^{n}\left(t_{n}\right) \cdots P_{\alpha_{1}}^{1}\left(t_{1}\right),
$$

7 Thanks are due to D. Craig for discussions of this example. 
and by $\widetilde{C}_{\alpha}$ the corresponding string of $C P T$-transformed alternatives written in standard time order with the earliest alternatives to the right

$$
\widetilde{C}_{\alpha} \equiv \widetilde{P}_{\alpha_{1}}^{1}\left(-t_{1}\right) \cdots \widetilde{P}_{\alpha_{n}}^{n}\left(-t_{n}\right)
$$

Thus,

$$
\widetilde{C}_{\alpha}=\Theta^{-1} C_{\alpha}^{\dagger} \Theta
$$

The requirement of $C P T$ symmetry is then, from (3.1),

$$
\operatorname{Tr}\left(\rho_{f} C_{\alpha} \rho_{i} C_{\alpha}^{\dagger}\right)=\operatorname{Tr}\left(\rho_{f} \widetilde{C}_{\alpha} \rho_{i} \widetilde{C}_{\alpha}^{\dagger}\right)
$$

Using (5.1), (5.3), the cyclic property of the trace, and the identity $\operatorname{Tr}\left[A \Theta^{-1} B \Theta\right]=$ $\operatorname{Tr}\left[B^{\dagger} \Theta A^{\dagger} \Theta^{-1}\right]$ following from the antiunitarity of $\Theta$, the right hand side of (5.4) may be rewritten to yield the following form of the requirement of CPT symmetry

$$
\operatorname{Tr}\left(\rho_{f} C_{\alpha} \rho_{i} C_{\alpha}^{\dagger}\right)=\operatorname{Tr}\left(\Theta \rho_{i} \Theta^{-1} C_{\alpha} \Theta \rho_{f} \Theta^{-1} C_{\alpha}^{\dagger}\right)
$$

Evidently a sufficient condition for a $C P T$-symmetric universe is that the initial and final conditions be $C P T$ transforms of each other:

$$
\rho_{f}=\Theta \rho_{i} \Theta^{-1}
$$

because acting on Bose operators $\Theta^{2}$ is effectively unity and as a consequence of (5.6) $\rho_{i}=\Theta \rho_{f} \Theta^{-1}$.

As stressed by Page [34], a CPT-symmetric universe can also be realized with within the usual formulation of quantum mechanics with an initial $\rho_{i}$ and a final $\rho_{f}=I$, provided the $\rho_{i}$ representing the condition at the initial instant is CPT-invariant about some time in the future. Thus, initial and final conditions that are not related by (5.6) do not necessarily imply differing probabilities for sets of histories connected by $C P T$. Further, as discussed in the previous section, both ways of realizing a $C P T$-symmetric universe can, with appropiate kinds of initial and final conditions and coarse-graining, lead to sets of histories in which each individual member is CPT-asymmetric about the moment of symmetry. Thus, neither are CPT-symmetric boundary conditions necessarily inconsistent with arrows of time that extend consistently over the whole of the universe's evolution.

A universe is time-symmetric about a moment of time if the probabilities of any set of alternative histories are identical to those of the time-inverted set. The relation between initial and final conditions necessary for a purely time-symmetric universe is analogous to that for a $C P T$-symmetric one and derived in the same way. However, we cannot expect boundary conditions to impose time symmetry if the Hamiltonian itself distinguishes past from future. We must assume that the Hamiltonian is symmetric under time inversion, $\mathcal{T}$,

$$
\mathcal{T}^{-1} H(t) \mathcal{T}=H(-t)
$$

Given (5.7), a time-symmetric universe will result if the initial and final conditions are related by time inversion:

$$
\rho_{f}=\mathcal{T} \rho_{i} \mathcal{T}^{-1}
$$


For realistic quantum cosmologies, the time-neutral quantum mechanics of universes in a box, described in Section III, must be generalized to allow for significant quantum fluctuations in spacetime geometry, and notions of space and time inversion must be similarly generalized. A sketch of a generalized quantum mechanics for spacetime can be found in 14] and discussions of time inversion in the quantum mechanics of cosmology in [34], [5], and [6].

\section{B. T Violation in the Weak Interactions}

The effective Hamiltonian describing the weak interaction on accessible energy scales is not $C P$-invariant. As a consequence of the $C P T$ invariance of field theory it is also not $T$ invariant. $T$ violation of this kind is a small effect in laboratory experiments but is thought to be of central importance in the evolution of the matter content of the universe. It is believed to be responsible, together with the non-conservation of baryons, for the emergence of a matter-dominated universe from an initial equality of matter and antimatter, as originally pointed out by Sakharov. ${ }^{8}$ Can the symmetric universes just discussed be consistent with this effective $T$ violation in the weak interaction?

The violation of time-inversion symmetry that we observe in the effective weak interaction Hamiltonian could arise in three ways: First, it could be the result of $T$ violation in the fundamental Hamiltonian. Second, it could arise throughout the universe even if the fundamental Hamiltonian were time-inversion-symmetric, from asymmetries in the cosmological boundary conditions of the universe. Third, it could be an asymmetry of our particular epoch and spatial location arising dynamically in extended domains from a time-inversion symmetric Hamiltonian and boundary conditions. We shall now offer a few comments on each of these possibilities.

If the fundamental Hamiltonian is time-inversion asymmetric, then we cannot expect a time-symmetric universe, as we have already discussed. One could investigate whether such a fundamental time asymmetry is the source of the other observed time asymmetries. So far such an approach has neither been much studied nor shown much promise.

Even though a $T$-symmetric universe is inconsistent with a $T$-asymmetric fundamental Hamiltonian, a CPT-symmetric universe could be realized if the initial and final density matrices were related by (5.6). That is because a field-theoretic Hamiltonian is always $C P T$-symmetric even if it is not $T$-symmetric. But $C P T$ symmetry needs to be reconciled with the observed matter-antimatter asymmetry over large domains ${ }^{9}$ of the universe and the classical behavior of their matter content. If the universe is homogeneously matter-dominated now, then CPT symmetry would imply that it will be homogeneously antimatter-dominated at the time-inverted epoch in the future. What evolution of the present universe could lead to such an inversion? One possibility is a universe that lasts much longer than the proton lifetime. ${ }^{10}$

\footnotetext{
${ }^{8}$ Ref. [36]. For an accessible recent review of these ideas see [37].

${ }^{9}$ For a classic review of the observational evidence that there is a matter-antimatter asymmetry over a domain at least the size of the local group of galaxies see [38].

${ }^{10}$ We owe this suggestion to W. Unruh.
} 
There is no evidence for $C P$ violation in the basic dynamics of superstring theory. If it is the correct theory, the effective $C P$ violation in the weak interaction in four dimensions has to arise in the course of compactification or from some other form of spontaneous symmetry breaking. From the four-dimensional point of view, which we are taking for convenience in this article, this would correspond to having a non-zero expected value of a $C P$-odd quantity. Then, as discussed above, it is possible to investigate time-symmetric universes with initial and final conditions related by (5.8). An effective $C P$ violation could arise from $C P$ asymmetries of the initial or final states or both. Typical theories of these boundary conditions relate them to the Hamiltonian or an equivalent action. Each density matrix, $\rho_{i}$ or $\rho_{f}$, may either inherit the symmetries of the fundamental Hamiltonian or be an asymmetrical member of a symmetrical family of density matrices determined by it. This is the case, for example, with "spontaneous symmetry breaking" of familiar field theory where there are degenerate candidates for the ground state not individually symmetrical under the symmetries of the Hamiltonian. Before discussing the possibility of effective $C P$ violation in time-symmetric universes, let us review how an effective $C P$ violation can arise in familiar field theory and in usual quantum cosmology with just an initial condition.

Effective $C P$ violation can arise in field theory even when the fundamental Hamiltonian is $C P$-invariant, provided there is a non-vanishing vacuum expected value of a $C P$-odd field $\phi(\vec{x}, t)$ [39], i.e. one such that

$$
\phi(-\vec{x}, t)=-(\mathcal{C P})^{-1} \phi(\vec{x}, t)(\mathcal{C P}) .
$$

Usually the vacuum state $\left|\Psi_{0}\right\rangle$ inherits the symmetry of the Hamiltonian that determines it and the vacuum expected value of a $C P$-odd field would vanish if the Hamiltonian is $C P$-invariant. However, if there is a symmetrical family of degenerate candidates for the ground state that are individually not $C P$-invariant, then the expected value

$$
\langle\phi(\vec{x}, t)\rangle=\operatorname{Tr}\left[\phi(\vec{x}, t)\left|\Psi_{0}\right\rangle\left\langle\Psi_{0}\right|\right]
$$

may be non-zero for the physical vacuum.

Similarly, in usual quantum cosmology with just an initial condition $\rho_{i}$, a non-zero value of

$$
\langle\phi(\vec{x}, t)\rangle=\operatorname{Tr}\left[\phi(\vec{x}, t) \rho_{i}\right]
$$

can lead to effective $C P$ violation. The "no-boundary" wave function of the universe 35] is the generalization of the flat space notion of ground state, i.e. vacuum, to the quantum mechanics of closed cosmological spacetimes. The "no-boundary" prescription with matter theories that would lead to spontaneous $C P$ violation in flat space thereby becomes an interesting topic for investigation. In such situations, we expect the "no-boundary" construction to yield a $C P$-symmetric set of possible wave functions for the universe that are individually $C P$-asymmetric.

We now turn to effective $C P$ violation in time-symmetric universes with initial and final states related by (5.8). An expected value for a field is defined when probabilities are assignable to its alternative values - that is, when there is decoherence among the alternatives. The requirements of decoherence will be discussed in the next Section. They are automatically satisfied for alternatives at a single moment of time when $\rho_{f} \propto I$ but they are non-trivial when $\rho_{f}$ is non-trivial. We have not analyzed the circumstances in which the 
values of the field decohere but we assume those circumstances to obtain here so that the expectation value of the field may be defined.

A consequence of decoherence and the probability formula (3.1) is the validity of two equivalent expressions for the expected value of the field that are analogous to (5.10) and (5.11):

$$
\langle\phi(\vec{x}, t)\rangle=N \operatorname{Tr}\left[\rho_{f} \phi(\vec{x}, t) \rho_{i}\right]=N \operatorname{Tr}\left[\rho_{i} \phi(\vec{x}, t) \rho_{f}\right] .
$$

These are demonstrated in the Appendix. The symmetry between the initial and final conditions in (5.12) can be understood from the fact that it is not probabilities at one moment of time that distinguish the future from the past. We shall now show that for a $C P$-odd field this expected value is odd under time inversion for a time-symmetric universe.

We carry over from flat space field theory the assumption that we are dealing with a $C P T$-even field $\phi(\vec{x}, t)$. In flat space that is necessary if the field is to have a non-vanishing vacuum expected value. The $C P T$ invariance of field theory then means that it is possible to choose a (real) representation of $\phi(\vec{x}, t)$ such that

$$
\phi(-\vec{x},-t)=(\mathcal{C P} \mathcal{T})^{-1} \phi(\vec{x}, t)(\mathcal{C P} \mathcal{T}) .
$$

Therefore, since $\phi(\vec{x}, t)$ is $C P$-odd it must be $T$-odd and then

$$
\langle\phi(\vec{x},-t)\rangle=-\operatorname{Tr}\left[\rho_{f} \mathcal{T}^{-1} \phi(\vec{x}, t) \mathcal{T} \rho_{i}\right] .
$$

But if $\rho_{i}$ and $\rho_{f}$ are related by (5.8) this relation may be written

$$
\begin{aligned}
\langle\phi(\vec{x},-t)\rangle & =-\operatorname{Tr}\left[\mathcal{T}^{-1} \rho_{i} \mathcal{T} \mathcal{T}^{-1} \phi(\vec{x}, t) \mathcal{T} \mathcal{T}^{-1} \rho_{f} \mathcal{T}\right] \\
& =-\operatorname{Tr}\left[\rho_{i} \phi(\vec{x}, t) \rho_{f}\right]=-\langle\phi(\vec{x}, t)\rangle .
\end{aligned}
$$

The conclusion is that it is possible to choose initial and final conditions so that a universe is time-symmetric and has a non-vanishing expected value of a $C P$-odd field. That expected value is odd in time (the correct time-symmetric behavior for a $T$-odd field.) As a consequence the sign of $C P$ violation would be opposite on opposite sides of the moment of time symmetry and the magnitude of $C P$ violation would decrease on cosmological time scales as the moment of time symmetry is approached. The $C P$ violation in the early universe might well be larger than generally supposed and Sakharov's mechanism for the generation of the baryons more effective. However, if the moment of time symmetry is far in our future, then such variation in the strength of $C P$ violation would be small and it would be difficult to distinguish this time-symmetric situation from the kind of $C P$ violation that arises from just an initial condition as discussed above.

In the class of time-symmetric universes just discussed, $C P$ violation arises from initial and final conditions that are not $C P$-symmetric. However, an effective $C P$ violation could also exist in our epoch, in local spatial domains, even if both Hamiltonian and initial and final states were $C P$-symmetric:

$$
H=(\mathcal{C P})^{-1} H(\mathcal{C P}), \rho_{i}=(\mathcal{C P})^{-1} \rho_{i}(\mathcal{C P}), \rho_{f}=(\mathcal{C P})^{-1} \rho_{f}(\mathcal{C P}) .
$$

Dynamical mechanisms would need to exist that make likely the existence of large spacetime domains in which $C P$ is effectively broken, say by the expected value of a $C P$-odd field that grows to be homogeneous over such a domain. In such a picture the set of histories of the 
universe would be overall $C P$-symmetric and $T$-symmetric, as follows from (5.16). Individual histories would display effective $C P$ violation in domains with sizes and durations that are quantum-probabilistic. If very large sizes and durations were probable it would be difficult to distinguish this kind of mechanism from any of those discussed above.

Overall matter-antimatter symmetry would be expected for such universes with matter or anti-matter predominant only in local domains. Their size must therefore be larger than the known scales on which matter is dominant [38]. The calculation of the probabilities for these sizes and durations thus becomes an important question in such pictures. An extreme example occurs in the proposal of Linde [40], in which such domains are far larger than the present Hubble radius.

\section{THE LIMITATIONS OF DECOHERENCE AND CLASSICALITY}

As we mentioned in Section II, the quantum mechanics of a closed system such as the universe as a whole predicts probabilities only for those sets of alternative histories for which there is negligible interference between the individual members in the set. Sets of histories that exhibit such negligible interference as a consequence of the Hamiltonian and boundary conditions are said to decohere. A minimal requirement on any theory of the boundary conditions for cosmology is that the universe exhibit a decoherent set of histories that corresponds to the quasiclassical domain of everyday experience. This requirement places significant restrictions on the relation between $\rho_{i}$ and $\rho_{f}$ in the generalized quantum mechanics for cosmology, as we shall now show.

\section{A. Decoherence}

Coherence between individual histories in an exhaustive set of coarse-grained histories is measured by the decoherence functional [20]. This is a complex-valued functional on each pair of histories in the set. If the cosmos is replaced by a box, so that possible complications from quantum gravity disappear, then individual coarse-grained histories are specified by sequences of alternatives $\alpha=\left(\alpha_{1}, \cdots, \alpha_{n}\right)$ at discrete moments of time, $t_{1}, \cdots, t_{n}$. The decoherence functional for the case of two-time boundary conditions is given by [15]

$$
D\left(\alpha^{\prime}, \alpha\right)=N \operatorname{Tr}\left[\rho_{f} C_{\alpha^{\prime}} \rho_{i} C_{\alpha}^{\dagger}\right]
$$

A set of histories decoheres when the real parts of the "off-diagonal" elements of the decoherence functional - those between two histories with any $\alpha_{k} \neq \alpha_{k}^{\prime}$ - vanish to sufficient accuracy. As first shown by Griffiths [13], this is the necessary and sufficient condition that the probabilities (3.1), which are the "diagonal" elements of $D$, satisfy the sum rules defining probability theory.

The possibility of decoherence is limited by the choice of initial and final density matrices $\rho_{i}$ and $\rho_{f}$. To see an example of this, consider the case in which both are pure, $\rho_{i}=\left|\Psi_{i}\right\rangle<$ $\Psi_{i} \mid$ and $\rho_{f}=\left|\Psi_{f}><\Psi_{f}\right|$. The decoherence functional would then factor:

$$
D\left(\alpha^{\prime}, \alpha\right)=N<\Psi_{f}\left|C_{\alpha^{\prime}}\right| \Psi_{i}><\Psi_{i}\left|C_{\alpha}\right| \Psi_{f}>
$$


where $N$ now is $\left|\left\langle\Psi_{i} \mid \Psi_{f}\right\rangle\right|^{-2}$. In this circumstance the requirement that the real part of $D$ vanish for $\alpha^{\prime} \neq \alpha$ can be satisfied only if there are at most two non-vanishing quantities $<\Psi_{i}\left|C_{\alpha}\right| \Psi_{f}>$, with phases differing by $90^{\circ}$, giving at most two histories with non-vanishing probabilities! Thus initial and final states that are both pure, such as those corresponding to a "wave function of the universe", leads to a highly unorthodox quantum mechanics in which there are only one or two coarse-grained histories. All the apparent accidents of quantum mechanics would be determined ${ }^{11}$ by the boundary conditions $\rho_{i}$ and $\rho_{f}$. The usual idea of a simple $\rho_{i}$ (or $\rho_{i}$ and $\rho_{f}$ ), with the algorithmic complexity of the universe contained almost entirely in the throws of the quantum dice, would here be replaced by a picture in which the algorithmic complexity is transferred to the state vectors $\left|\Psi_{i}\right\rangle$ and $\left|\Psi_{f}\right\rangle$. Presumably these would be described by a simple set of rules plus a huge amount of specific information, unknowable except by experiment and described in practice by a huge set of parameters with random values.

This bizarre situation refers to the use of a pure $\rho_{i}$ and a pure $\rho_{f}$, whether or not there is any kind of time symmetry relating them.

\section{B. Impossibility of a Universe with $\rho_{f}=\rho_{i}$.}

We shall now give a very special example of a relation between $\rho_{i}$ and $\rho_{f}$, stronger than time symmetry, that is inconsistent with the existence of a quasiclassical domain. More precisely we shall show that in the extreme case

$$
\rho_{f}=\rho_{i} \equiv \rho
$$

only sets of histories exhibiting trivial dynamics can exactly decohere. This condition means that $\rho_{f}$ has the same form when expressed in terms of the initial fields $\phi\left(\vec{x}, t_{0}\right)$ as $\rho_{i}$ does. Such a situation could arise if, in addition to time symmetry, we had $\rho_{i}$ and $\rho_{f}$ separately, individually time-symmetric and with effectively no time difference between the initial and final conditions. We know of no theoretical reason to expect such a situation, but it does supply an example that leads to a contradiction with experience.

Given the artificial condition, (6.3), we can write the decoherence condition as

$$
\left(\operatorname{Tr} \rho^{2}\right)^{-1} \operatorname{Re} \operatorname{Tr}\left(\rho C_{\alpha^{\prime}} \rho C_{\alpha}^{\dagger}\right)=\delta_{\alpha^{\prime} \alpha} p(\alpha)
$$

where $p(\alpha)$ is the probability of the history $\alpha$. Summing over all the $\left\{\alpha_{n}\right\}$ and $\left\{\alpha_{n}^{\prime}\right\}$ except $\alpha_{k}$ and $\alpha_{k}^{\prime}$, we have

$$
\left(\operatorname{Tr} \rho^{2}\right)^{-1} \operatorname{Re} \operatorname{Tr}\left[\rho P_{\alpha_{k}^{\prime}}^{k}\left(t_{k}\right) \rho P_{\alpha_{k}}^{k}\left(t_{k}\right)\right]=\delta_{\alpha_{k}^{\prime} \alpha_{k}} p\left(\alpha_{k}\right)
$$

We note that $P_{\alpha_{k}}^{k}\left(t_{k}\right)$ and $P_{\alpha_{k}^{\prime}}^{k}\left(t_{k}\right)$ are just projection operators and thus of the form

$$
\sum_{n}|n><n| \text { and } \sum_{n^{\prime}}\left|n^{\prime}><n^{\prime}\right|
$$

11 This situation is closely related to the one described by L. Schulman 41]. 
respectively, where the $\mid n>$ and $\mid n^{\prime}>$ are mutually orthogonal for $\alpha_{k} \neq \alpha_{k}^{\prime}$. Eq. (6.5) then tells us that

$$
\left(\operatorname{Tr} \rho^{2}\right)^{-1} \sum_{n, n^{\prime}}|<n| \rho\left|n^{\prime}>\right|^{2}=0 \text { for } \alpha_{k} \neq \alpha_{k}^{\prime} .
$$

Thus $\rho$ has no matrix elements between any $\mid n>$ and any $\mid n^{\prime}>$ for $\alpha_{k} \neq \alpha_{k}^{\prime}$. In other words, $\rho$ commutes with all the $P$ 's and therefore with all the chains $C_{\alpha}$ of $P$ 's:

$$
\left[C_{\alpha}, \rho\right]=0 \text { for all } \alpha \text {. }
$$

This consequence of perfect decoherence for the special case (6.3) has some important implications. For one thing, the decoherence formula can now be written

$$
\left(\operatorname{Tr} \rho^{2}\right)^{-1} \operatorname{Tr}\left(C_{\alpha^{\prime}} \rho^{2} C_{\alpha}^{\dagger}\right)=\delta_{\alpha^{\prime} \alpha} p(\alpha)
$$

so that we are back to ordinary quantum mechanics with only an initial density matrix $\bar{\rho} \equiv\left(\operatorname{Tr} \rho^{2}\right)^{-1} \rho^{2}[c f .(4.5)$ in the classical case] but with the very restrictive condition

$$
\left[C_{\alpha}, \bar{\rho}\right]=0 \text { for all } \alpha \text {. }
$$

The cosmology with the symmetry (6.3) was supposed to be in contrast to the usual one with only an initial density matrix, and yet it turns out to be only a special case of the usual one with the stringent set of conditions (6.9) imposed in addition. The resolution of this apparent paradox is that Eq. (6.9) permits essentially no dynamics and thus achieves symmetry between $\rho_{i}$ and $\rho_{f}$ in a rather trivial way. That is not surprising in view of the nature of this condition discussed above.

We have seen that any $P_{\alpha_{k}}^{k}\left(t_{k}\right)$ has to commute with $\bar{\rho}$ if it is to be permitted in a chain of $P$ 's constituting a member of a decohering set of alternative coarse-grained histories. Now it is unreasonable that for a given projection operator $P$ there should be only a discrete set of times at which it is permissible to use it in a history (e.g., for a measurement). Thus we would expect that there should be a continuous range of such times, which means that $\dot{P}=-i[P, H]$ must commute with $\bar{\rho}$. But $\bar{\rho}$ and $P$, since they commute, are simultaneously diagonalizable, with eigenvalues $\pi_{i}$ and $q_{i}$ respectively. The time derivative of the probability $\operatorname{Tr}(\bar{\rho} P)$ is

$$
\operatorname{Tr}(\bar{\rho} \dot{P})=-i \operatorname{Tr}(\bar{\rho}[P, H])=-i \sum_{i} \pi_{i}\left(q_{i}-q_{i}\right) H_{i i}=0 .
$$

The probabilities of the different projections $P$ remain constant in time, so that there is essentially no dynamics and certainly no second law of thermodynamics.

\section{Classicality}

A theory of the boundary conditions of the universe must imply the quasiclassical domain of familiar experience. A set of histories describing a quasiclassical domain must, of course, decohere. That is the prerequisite for assigning probabilities in quantum mechanics. But further, the probabilities must be high that these histories are approximately correlated by classical dynamical laws, except for the intervention of occasional amplified quantum fluctuations. 
There are, of course, limitations on classical two-time boundary conditions. We cannot, for example, specify both coördinates and their conjugate momenta at both an initial and a final time. There would, in general, be no corresponding solutions of the classical equations of motion. Even if initial and final conditions in quantum cosmology allow for decoherence as discussed above, they could still be too restrictive to allow for classical correlations. One would expect this to be the case, for example, if they required a narrow distribution of both coordinates and momenta both initially and finally. Quantum cosmologies with two boundary conditions are therefore limited by both decoherence and classicality.

\section{CONCLUSIONS}

Time-symmetric quantum cosmologies can be constructed utilizing a time-neutral generalized quantum mechanics of closed systems with initial and final conditions related by time-inversion symmetry. From the point of view of familiar quantum mechanics such timesymmetric cosmologies are highly unusual. If we think of Hilbert space as finite-dimensional, we could introduce a normalization $\operatorname{Tr}\left(\rho_{f}\right) \operatorname{Tr}\left(\rho_{i}\right)=\operatorname{Tr}(I)$, which would agree with the usual case $\operatorname{Tr}\left(\rho_{i}\right)=1, \operatorname{Tr}\left(\rho_{f}\right)=\operatorname{Tr}(I)$. (Note that both $N=\operatorname{Tr}\left(\rho_{f} \rho_{i}\right)$ and the quantity $\operatorname{Tr}\left(\rho_{f}\right) \operatorname{Tr}\left(\rho_{i}\right)$ are invariant under multiplication of $\rho_{i}$ by a factor and $\rho_{f}$ by the inverse factor.) With this normalization we may think of $N^{-1}=\operatorname{Tr}\left(\rho_{f} \rho_{i}\right)$ as a measure of the likelihood of the final condition given the initial one. The similarly defined quantity $N^{-1}$ in the analogous classical time-symmetric cosmologies is just that. It is the fraction of trajectories meeting the initial condition that also meet the final one [cf. (4.1)]. The measure $N^{-1}$ is unity for the usual cases where $\rho_{f}=I$. It can be expected to be very small for large systems with time-symmetric boundary conditions, as the simple model described in Figure 1 suggests. The measure $N^{-1}$ is likely to be extraordinarily small in the case of the universe itself. Were it exactly zero the initial and final boundary condition construction would become doubtful. We are unsure how much of that doubt survives if it is merely extraordinarily small.

As a prerequisite for a time-symmetric quantum cosmology, the fundamental Hamiltonian must be time-inversion symmetric to give a meaningful notion of time-symmetry and this restricts the mechanisms by which the effective $C P$ violation in the weak interactions can arise. There must be some impurity in the initial or final density matrices or in both for any non-trivial probabilities to be predicted at all. If we wish to exclude the highly unorthodox quantum mechanics in which $\left|\Psi_{i}\right\rangle$ and $\left|\Psi_{f}\right\rangle$ determine all the throws of the quantum dice, then we could not have, for example, a time-symmetric quantum cosmology with both the initial and final conditions resembling something like the "no-boundary" proposal. These results have been obtained by assuming unrealistic exact decoherence and by neglecting gross quantum variations in the structure of spacetime, which may be important in the early universe. It would be desirable to extend the discussion to remove these special restrictions.

Even if these purely theoretical requirements for time-symmetry were met, observations might rule out such boundary conditions. Deviations from the usual thermodynamic or $C P$ arrows of time may be undetectably small if the time between initial and final conditions is long enough. But, as suggested by Davies and Twamley, an expanding and contracting time-symmetric cosmology may be transparent enough to electromagnetic and other forms of radiation that the effects of time-symmetric initial and final conditions would be inconsistent 
with observations today. In the absence of some compelling theoretical principle mandating time symmetry, the simplest possibility seems to be the usually postulated universe where there is a fundamental distinction between past and future - a universe with a special initial state and a final condition of indifference with respect to state. Nevertheless, the notion of complete $T$ symmetry or $C P T$ symmetry remains sufficiently intriguing to warrant further investigation of how such a symmetry could occur or what observations could rule it out. In this paper we have provided a quantum-mechanical framework for such investigations.

\section{Acknowledgments}

An earlier version of this paper appeared in the Proceedings of the 1st International Sakharov Conference on Physics, Moscow, USSR, May 27-31, 1991 as a tribute to the memory of A.D. Sakharov.

Part of this research was carried out at the Aspen Center for Physics. The work of MG-M was supported by DOE contract DE-AC-03-81ER40050 and by the Alfred P. Sloan Foundation. That of JBH was supported by NSF grant PHY90-08502.

\section{Appendix}

We derive the expression (5.12) for the expected value of a scalar field in the time-neutral quantum mechanics of cosmology with an initial condition represented by a density matrix $\rho_{i}$ and a final condition represented by a density matrix $\rho_{f}$. Consider alternatives such that the value of the field $\phi$ at $(\vec{x}, t)$ lies in one of an exhaustive set of infinitesimal exclusive intervals $\left\{\Delta_{\alpha}\right\}$ with central values $\left\{\phi_{\alpha}\right\}$. Let $P_{\alpha}(\vec{x}, t)$ denote the corresponding projection operators. The decoherence functional for this set of alternatives is, according to (6.1)

$$
D\left(\alpha^{\prime}, \alpha\right)=N \operatorname{Tr}\left[\rho_{f} P_{\alpha^{\prime}}(\vec{x}, t) \rho_{i} P_{\alpha}(\vec{x}, t)\right] .
$$

We assume that this is diagonal, that is, proportional to $\delta_{\alpha \alpha^{\prime}}$. The diagonal elements give the probabilities of the alternative values of the field according to (3.1). Thus the expected value of $\phi(\vec{x}, t)$ is

$$
\langle\phi(\vec{x}, t)\rangle=\sum_{\alpha} \phi_{\alpha} D(\alpha, \alpha) .
$$

Because the alternatives decohere, this can be written in two equivalent forms

$$
\langle\phi(\vec{x}, t)\rangle=\sum_{\alpha^{\prime} \alpha} \phi_{\alpha^{\prime}} D\left(\alpha^{\prime}, \alpha\right)=\sum_{\alpha^{\prime} \alpha} \phi_{\alpha} D\left(\alpha^{\prime}, \alpha\right) .
$$

But, utilizing $\sum_{\alpha} P_{\alpha}(\vec{x}, t)=1$ and $\sum_{\alpha} \phi_{\alpha} P_{\alpha}(\vec{x}, t)=\phi(\vec{x}, t)$, as well as (A.1) and the cyclic property of the trace, we get

$$
\langle\phi(\vec{x}, t)\rangle=N \operatorname{Tr}\left[\rho_{f} \phi(\vec{x}, t) \rho_{i}\right]=N \operatorname{Tr}\left[\rho_{i} \phi(\vec{x}, t) \rho_{f}\right]
$$

as in (5.12).

[1] P.C.W. Davies (1976) The Physics of Time Asymmetry, University of California Press, Berkeley. 
[2] R. Penrose (1979) in General Relativity: An Einstein Centenary Survey ed. by S.W. Hawking and W. Israel, Cambridge University Press, Cambridge, UK.

[3] H.D. Zeh (1989) The Physical Basis of the Direction of Time, Springer, Berlin.

[4] J.J. Loschmidt (1876) Wiener Ber. 73, 128; (1877) ibid. 75, 67.

[5] D. Page (1985) Phys. Rev. D 32, 2496.

[6] S.W. Hawking (1985) Phys. Rev. D 32, 2989.

[7] J. Halliwell and S.W. Hawking (1985) Phys. Rev. D 31, 1777.

[8] R. Laflamme (Unpublished) "Wave Function of an $S^{1} \times S^{2}$ Universe".

[9] S.W. Hawking (1987) New Scientist 115, 46.

[10] S.W. Hawking and D. Page (1988) Nucl. Phys. B 298, 789.

[11] S. Wada (1990) in Proceedings of the 3rd International Symposium on the Foundations of Quantum Mechanics in the Light of New Technology, ed. by S. Kobayashi, H. Ezawa, Y. Murayama, and S. Nomura, Physical Society of Japan, Tokyo.

[12] Y. Aharonov, P. Bergmann, and J. Lebovitz (1964) Phys. Rev. B 134, 1410.

[13] R.B. Griffiths (1984) J. Stat. Phys. 36, 219.

[14] J.B. Hartle (1991) in Quantum Cosmology and Baby Universes, Proceedings of the 1989 Jerusalem Winter School, ed. by S. Coleman, J. Hartle, T. Piran, and S. Weinberg, World Scientific, Singapore.

[15] T. Gold (1958) in La structure et l'evolution de l'universe, Proceedings of the 11th Solvay Congress, Editions Stoops, Brussels; (1962) Amer. J. Phys. 30, 403.

[16] M. Gell-Mann (unpublished) comments at the 1967 Temple University Panel on Elementary Particles and Relativistic Astrophysics.

[17] Y. Ne'eman (1970) Int. J. Theor. Phys. 3, 1.

[18] H.-D. Zeh (1993) in Proceedings of the NATO Workshop on the Physical Origins of Time Asymmetry, ed. by J. Halliwell, J. Perez-Mercader, and W. Zurek, Cambridge University Press, Cambridge, UK.

[19] S.W. Hawking (1993) in Proceedings of the NATO Workshop on the Physical Origins of Time Asymmetry, ed. by J. Halliwell, J. Perez-Mercader, and W. Zurek, Cambridge University Press, Cambridge, UK.

[20] M. Gell-Mann and J.B. Hartle (1990) in Complexity, Entropy, and the Physics of Information, SFI Studies in the Sciences of Complexity, Vol. VIII, ed. by W. Zurek, Addison Wesley, Reading or in Proceedings of the 3rd International Symposium on the Foundations of Quantum Mechanics in the Light of New Technology ed. by S. Kobayashi, H. Ezawa, Y. Murayama, and S. Nomura, Physical Society of Japan, Tokyo.

[21] M. Gell-Mann and J.B. Hartle (1990) in the Proceedings of the 25th International Conference on High Energy Physics, Singapore, August, 2-8, 1990, ed. by K.K. Phua and Y. Yamaguchi, South East Asia Theoretical Physics Association and Physical Society of Japan, distributed by World Scientific, Singapore.

[22] W.J. Cocke (1967) Phys. Rev. 160, 1165.

[23] L.S. Schulman (1973) Phys. Rev. D 7, 2868; L.S. Schulman (1977) J. Stat. Phys. 16, 217; L.S. Schulman and R. Shtokhamer (1977) Int. J. heor. Phys. 16, 287.

[24] J.A. Wheeler (1979) in Problemi dei fondamenti della fisica, Scuola internazionale di fisica "Enrico Fermi", Corso 52, ed. by G. Toraldo di Francia, North-Holland, Amsterdam.

[25] L.S. Schulman (to appear in the M. Fisher Festschrift)

[26] M. Kac (1959) Probability and Related Topics in Physical Sciences, Interscience, New York.

[27] R. Penrose (1978) in Confrontation of Cosmological Theories with Observational Data (IAU 
Symp. 63) ed. by M. Longair, Reidel, Boston (1974) and in Theoretical Principles in Astrophysics and Relativity, ed. by N.R. Lebovitz, W.H. Reid, and P.O. Vandervoort, University of Chicago Press, Chicago (1978).

[28] L. Grishchuk and L.V. Rozhansky (1988) Phys. Lett. B 208, 369; (1990) ibid. 234, 9.

[29] A. Barvinsky and A. Kamenshchik (1990) Class. Quant. Grav. 7, L181.

[30] A. Vilenkin (1988) Phys. Rev. D 37, 888.

[31] M. Mijić, M. Morris and W.-M. Suen (1989) Phys. Rev. D 39, 1486.

[32] P.C.W. Davies and J. Twamley, (1993) Class. Quant. Grav. 10, 931.

[33] R.B. Partridge (1973) Nature 244, 263.

[34] D. Page (1993), No Time Asymmetry from Quantum Mechanics, University of Alberta preprint.

[35] J.B. Hartle and S.W. Hawking (1983) Phys. Rev. D 28, 2960.

[36] A.D. Sakharov (1967) ZhETF Pis'ma 5, 32; (1967) Sov. Phys. JETP Lett. 5, 24; (1979) ZhETF 76, 1172, 1979; [(1979) Sov. Phys. JETP 49, 594.

[37] E.W. Kolb and M.S. Turner (1990) The Early Universe, Addison-Wesley, Redwood City, Ca.

[38] G. Steigman (1976) Ann. Rev. Astron. Astrophys. 14, 339.

[39] T.D. Lee (1974) Physics Reports 9C, 144.

[40] A. Linde (1986) Mod. Phys. Lett. A 1, 81; (1987) Physica Scripta T15, 169.

[41] L. Schulman (1986) J. Stat. Phys. 42, 689.

[42] J.P. Paz and W. Zurek, Phys. Rev. D, 48, 2728, 1993.

[43] M. Gell-Mann and J.B. Hartle, Phys. Rev. D, 47, 3345, 1993. gr-qc/9210010.

[44] J. Finkelstein (1993), Phys. Rev. D 47, 3345.

[45] M. Gell-Mann and J.B. Hartle, Strong Decoherence, to be published in the Proceedings of the 4th Drexel Symposium on Quantum Non-Integrability - The Quantum-Classical Correspondence, Drexel University, September 8-11, 1994, ed. by. D.-H. Feng and B.-L. Hu, International Press, Boston/Hong-Kong; gr-qc/9509054.

\section{Questions and Answers}

B. DeWitt: If you propose that the universe is in a particular quantum state, determined by particular initial conditions, why do you bother with a complete Hilbert-space framework for discussion?

JH: I interpret your question as mainly referring to the status of the superposition principle in quantum cosmology. It is true that if the initial condition of our universe is described by a single wave function then it is never necessary to superpose it with another to make predictions. However, the principle of superposition enters centrally elsewhere in the predictive framework. Specifically, it enters into the construction of the probabilities for the coarse-grained sets of histories that we observe. If $P_{A}$ and $P_{B}$ are projection operators representing exclusive alternatives $A$ and $B$ at one time, then the alternative $A$ and $B$ is represented by the sum of the projections, $P_{A}+P_{B}$. That is a specific instance of the principle of superposition. More generally, the decoherence functionals for sets of histories related by an operation of coarse graining must be connected by the superposition principle. That is one reason we assume the full apparatus of Hilbert space when discussing the quantum cosmology of matter fields in a fixed background spacetimes or generalizations of 
that formalism consistent with the superposition principle when quantum gravity is taken into account. Even in the most general cosmological context it should still be possible to test these aspects of the principle of superposition.

K. Kuchař: Both Murray's and your talk were based on the assumption that there is a true Hamiltonian and that there is a single time parameter which orders the projection operators. How does one formulate the difference between time-symmetric initial and final conditions if the dynamics is driven by constraints and there is no privileged time parameter?

JH: To keep the discussion in the talk manageable, we assumed a fixed, background spacetime. That, of course, is an excellent approximation anytime much more than a Planck time after the initial singularity and a Planck time before the final singularity if there is one. That fixed spacetime geometry supplies the notion of time used to order the operators and define the Hamiltonian. However, in regimes near the singularity, where quantum gravity is important and the geometry of spacetime fluctuates quantum mechanically, there will be no fixed spacetime geometry to supply a notion of time. A further generalization of quantum mechanics is thus required. I have described in several places the basic elements of one such generalization based on sum-over-histories quantum mechanics. ${ }^{12}$ In that generalization, the histories are four dimensional cosmological spacetimes with boundaries where the analogs of "initial" and "final" conditions represented by wave functions are imposed. The decoherence functional for coarse-grained sets of alternative histories of the universe, including diffeomorphism invariant coarse grainings of spacetime geometry, is represented in a sum-over-histories form that does not single out a privileged time parameter. Decoherence is thus defined and probabilities for the individual members of decoherent sets of coarse-grained histories can be calculated. In order not to be manifestly inconsistent with observations, the specific initial and final conditions of our universe had better predict the approximately classical behavior of spacetime geometry on accessible scales in our epoch. That is, semiclassically, realistic boundary conditions predict an ensemble of possible classical spacetimes of which we live in one. The probabilities of suitably coarse-grained matter field histories in each spacetime in the ensemble would be approximately given by the kind of quantum mechanics we have limited ourselves to in this talk with a notion of time given by the particular classical spacetime geometry. The discussion we gave is thus both a model for the more general case of quantum gravity and an approximation to it in all directly accessible circumstances.

If the initial and final conditions are suitably related, I would expect the ensemble of possible spacetimes predicted semiclassically by such a theory to exhibit statistical time symmetry. Further, for the probable spacetimes that are time symmetric I would expect the quantum mechanics of matter fields and small fluctuations of geometry to be time-

${ }^{12}$ See, for example, my lectures "The Quantum Mechanics of Cosmology" in Quantum Mechanics and Baby Universes: Proceedings of the 1989 Jerusalem Winter School, edited by S. Coleman, J. Hartle, T. Piran, and S. Weinberg, World Scientific, Singapore, 1990, or in more complete detail, in my lectures "Spacetime Quantum Mechanics and the Quantum Mechanics of Spacetime" in Gravitation and Quantizations, Proceedings of the 1992 Les Houches Summer School, ed. by B. Julia and J. Zinn-Justin, North Holland, Amsterdam, 1993. 
symmetric in the sense have described in this talk. Put briefly, I expect the present discussion that assumes a fixed spacetime is a good approximation in interesting circumstances to the more general case where it is allowed to fluctuate. It is fair to say, however, that detailed calculations have not been done to check on these expectations.

R. Omnés: Your probability formula, in a universe with a destiny, violates Gleason's theorem and therefore one of its assumptions at least. There are two possibilities: (i) Not all properties are possible, which is what you are aiming at. (ii) Maybe, the Hilbert space is highly non-separable.

\section{JH: I think it's the former.}

J. Halliwell: You argue that for initial and final density matrices satisfying a certain condition (and in particular, for pure initial and final states) the decoherence functional factors, and therefore, will not decohere except for certain trivial histories. You suggested that there will therefore be problems for the no-boundary state, which is a pure state. It seems to me that this result may depend rather crucially on the existence of a Hilbert space structure etc., and in particular, on the possibility of folding in initial and final states using the usual inner product. My point is that all of this structure is not known to exist for quantum cosmology. The decoherence functional for quantum cosmology is yet to be constructed, and is likely to have a structure rather different to the quantum mechanical one. You may therefore be premature in your conclusions about the no-boundary proposal.

$\mathrm{JH}$ : In the proposals for the decoherence functional for quantum cosmology that I have put forward, the result that pure initial and final states permit the decoherence of only trivial sets of histories continues to hold in much the same way it does for the quantum cosmologies in a box described in the text. Initial and final conditions are represented by density matrices and pure conditions by single wave functions. The principle of superposition of amplitudes is maintained as is the relation between amplitudes and probabilities. When the initial and final states are pure the decoherence functional factors into a term for one history times a term for the other as in (6.2) and the rest of the argument goes through. In these generalizations imposing the "no boundary" proposal for both initial and final conditions does not lead to interesting sets of decohering histories. However, there is much to be investigated here and there could be other generalizations of quantum mechanics for which the result does not hold.

I. Bialynicki-Birula: I would like to make a comment on the possible role of soft photons in the time-symmetric quantum theory. In the presence of massless particles, and that is a typical case, even for time-symmetric Hamiltonians there is a difficulty in implementing the time-symmetry condition,

$$
\rho_{f}=\mathcal{T}^{-1} \rho_{i} \mathcal{T}
$$

due to the existence of infrared radiation. There is no $\rho_{f}$ that will give a nonvanishing transition probability whenever charged particles are being accelerated. In order to obtain a finite result we must perform an integration over the momenta of final, unobservable soft photons. The necessity to perform this integration implies that there is an asymmetry 
between the initial state described by $\rho_{i}$ and the final state for which a density operator does not exist.

JH: Soft photons in the universe certainly provide an important and widespread mechanism for decoherence. However, I haven't thought through the effect soft photons might have on the size of the normalizing factor $N^{-1}=\operatorname{Tr}\left(\rho_{f} \rho_{i}\right)$ that occurs in the expression (3.1) for probabilities. I would be surprised if, in a proper formulation of quantum electrodynamics, $N^{-1}$ necessarily vanished identically for the finite (although cosmologically long) time interval between time-symmetric initial and final conditions that we have been discussing. However, even if $N^{-1}$ does not vanish identically, but is only very small, that would signal a significant difference between the statistics of histories in a time-symmetric universe and the usual case. One guesses that $N^{-1}$ is likely to be small in any realistic time-symmetric even in the absence of electrodynamics but if soft photons play a significant role in determining its size that would be very interesting.

D. Page: What are the consequences if you require a $C P T$-invariant universe instead of a T-invariant universe?

JH: I didn't get to CPT-symmetric cosmologies in my talk, but they are discussed in the written contribution that Murray Gell-Mann and I have submitted to the proceedings. In the generalization of quantum mechanics that we discuss, a $C P T$-symmetric universe will result if the initial and final density matrices are related by a $C P T$ transformation. Since all local field theories are $C P T$-invariant, $C P T$-symmetric cosmologies are possible even if the $C P$ violation observed in the weak interactions arises from a fundamental Hamiltonian that is $C P$-non-invariant. That is in contrast to the case of $\mathrm{T}$ symmetry which can only be achieved with a $C P$-symmetric Hamiltonian so that the observed $C P$ violation must arise from one of the symmetry breaking mechanisms we discussed. However, also as discussed in the text, CPT symmetry may be difficult to reconcile with universes that are homogeneously dominated by matter near one singularity (and therefore antimatter dominated near the other) unless the lifetime is very long.

B. DeWitt: So if the no boundary condition leads to a $C P$ invariant family of $C P$ violating states, you must pick one member of this family out by hand?

JH: If the "no boundary" condition leads to a $C P$ invariant family of $C P$ non-invariant states then I would prefer to say that the initial condition is a density matrix with probabilities distributed uniformly among these possibilities. But for predictive purposes that amounts to what you said.

A. Albrecht: If one discusses the thermodynamic arrow of time in a time symmetric universe, one has a region near the "beginning" where the arrow runs toward the middle, and a region near the "end" where the arrow runs towards the middle. In the middle there is no particular arrow of time. The probability that we survive into the future epoch where the arrow is reversed is no greater than the probability that some IGUS is present right now, evolving in the opposite direction of time. 
JH: I think that's essentially right with a few qualifications. It follows from the assumed time symmetry that the statistics of IGUS's at the present age from the big bang must be the same as that at a comparable time from the final singularity if the only input to estimating those statistics is the initial and final conditions. If the lifetime of the universe is long compared to those times both the probability that an IGUS survives into the far future and the probability that there are IGUSes in the present living backward in time may be very low. If we are a typical IGUS then those probabilities apply to us. However, we have more information about our particular history with which the conditional probabilities of our surviving into the far future could, in principle, be calculated and compared with the probability that there are IGUSes that have evolved backward from the far future around today. I'll leave it to you to make the estimate of whether our particular history makes it more or less probable that we survive farther into the future than the typical IGUS!

P. Davies: Your model is most plausible if all asymmetric physical processes relax to equilibrium before the time reversal occurs. But long ago it was found that the future light cone in Robertson-Walker cosmological models is transparent to photons. (In the recontracting phase it is transparent at least until the turnover point.) Thus, retarded radiation cannot equilibrate before time reversal, so that the imposing of time-symmetric boundary conditions would surely show up experimentally in the emission of radiation.

JH: As mentioned in the talk, there are several different examples of physical systems that will not come to equilibrium in the Hubble time, and the system of matter and radiation is one of them. I take your comment to be a suggestion that observation in the electromagnetic system could supply the best lower bound on the time between initial and final conditions beyond which these are indistinguishable. That may well be the case and is an interesting subject for further research. It's an important question. [For further discussion see Section IVc, added after the conference.]

A. Starobinsky: (in response to P. Davies' comment)

This is not always the case. If the width of a domain wall in flat spacetime is larger than the radius of curvatures "gravitational radius" corresponding to the field energy density in a metastable state at the top of the field potential, then regions with the different sign of $C P$ violation are always beyond an observer's particle horizon and they are never accessible to him for any future evolution. A good example can be constructed using the "new" inflationary scenario and relating the sign of an inflation field to the sign of $C P$ violation.

\section{Note added by M Gell-Mann and J.B. Hartle, November 1993.}

We would like to clear up any possible confusion over the relationship among several ideas in the quantum mechanics of closed systems as well as the history of the subject and the terminology employed. In this note, added two years after the discussion, we attempt to clarify these matter as we see them, benefiting from research in the intervening time.

As far as we are aware, in the context of the modern interpretation of quantum mechanics, the term "decoherence" was first used by us in lectures and discussion after 1986 (summarized in our paper published in 1990) to describe a property of a set of alternative 
time-histories of a closed system. Specifically a set of histories is said to (medium) decohere when there is negligible or vanishing quantum mechanical interference between the individual histories in the set as measured by the "off-diagonal" elements of the decoherence functional $D\left(\alpha^{\prime}, \alpha\right)$. However, the term "decoherence" has subsequently come to be used to refer also to the decay over time of the off-diagonal elements of a reduced density matrix defined by a division of a complete set of variables into ones that are traced over in constructing the reduced density matrix and the rest. The decay was discussed in connection with the interpretation of quantum mechanics in the '70s and early '80s by Zeh, Zurek, and others (although not referred to as decoherence) and by many others since. These two notions of decoherence - of histories and of density matrices - are not the same but are not unconnected either. The vanishing at a sequence of times of the off-diagonal elements of a reduced density matrix in certain variables is neither mathematically nor physically equivalent to the decoherence of the corresponding set of alternative histories. However, the ideas are connected in certain idealized models where it can be shown that the physical mechanisms causing the decoherence of histories coarse-grained by ranges of values of certain sets of coordinates suitably spaced in time, also lead to the diagonalization of the reduced density matrix in these variables over similar intervals of time [14]. It has also been shown that in similar models, under restrictive conditions, the diagonalization of the reduced density matrix implies the decoherence of the histories associated with the "Schmidt basis" for that density matrix at suitable times 42, 43]. Finally, a certain interpretation of "mechanism of decoherence" can be defined [44, 45] that generalizes the reduced density matrix concept of decoherence in the context of a stronger form of decoherence of histories. A precise connection is thereby established between the two kinds of decoherence. The reader should therefore keep in mind that in these and other discussions and papers at this conference the word "decoherence" is used for two distinct but connected ideas - the decoherence of reduced density matrices and the decoherence of histories.

We now try to clarify the connection of our ideas with the work of Bob Griffiths and Roland Omnès, another topic that was raised in the discussion. In any quantum mechanical theory, a rule is needed to discriminate between those sets of histories that can be assigned probabilities and those that cannot because of quantum mechanical interference. Griffiths was the first to propose a quantum mechanics of closed systems with a rule that did not involve a fundamental notion of measurement. Instead, probabilities are assigned to just those sets of histories that are "consistent" in the sense that their probabilities obey correct sum rules - essentially the consistency requirement that the theory not offer two different results for the same probability. (In this connection, it may be helpful to note that what is sometimes referred to as the quantum mechanics of an "open system" means a set of effective rules for describing the quantum mechanical behavior of part of a closed system.) Griffiths' ideas were extended by Omnès and a similar formulation was arrived at independently, but later, by ourselves. As far as we are aware, all formulations of the quantum mechanics of closed systems under serious consideration, including ours, are "consistent history formulations" in the sense of requiring the consistency of a set of probability sum rules for those histories that are assigned probabilities. However, there are different formulations depending on just what probability sum rules are required and the strength of the conditions used to ensure their consistency.

The consistency conditions of Griffiths are not the same as the decoherence conditions used in our work. To explain the difference, a small amount of notation is useful. Let 
$\left\{C_{\alpha}\right\}$ denote a set of chains of projections representing a set of alternative histories, and $D\left(\alpha^{\prime}, \alpha\right)=\operatorname{Tr}\left(C_{\alpha^{\prime}} \rho C_{\alpha}\right)$ the associated decoherence functional. The consistency condition of Griffiths is that $\operatorname{Re} D\left(\alpha^{\prime}, \alpha\right) \approx 0, \alpha^{\prime} \neq \alpha$, if and only if $C_{\alpha^{\prime}}+C_{\alpha}$ is another chain of projections. This is the necessary condition for the probability sun rules if only histories corresponding to independent sets of alternatives at different moments of time (histories which are represented by chains of projections) are allowed. What we called the weak decoherence condition, $\operatorname{Re} D\left(\alpha^{\prime}, \alpha\right) \approx 0, \alpha^{\prime} \neq \alpha$, is stronger than that of Griffiths. It is the necessary condition if histories that are sums of chains of projections are allowed (corresponding to a rule for coarse-graining that allows arbitrary unions of histories as new histories). Our medium decoherence condition, $D\left(\alpha^{\prime}, \alpha\right) \approx 0, \alpha^{\prime} \neq \alpha$, is still stronger, implying both of the above conditions but not being implied by either of them. Thus, there are at least three different notions of decoherence of histories, all of which imply the consistency of (sometimes different) sets of probability sum rules.

In our work we have sought a notion of decoherence of histories that would capture generally the idea that, in physically interesting situations, relevant for quasiclassical behavior, quantum mechanical interference between histories vanishes for a reason. That is, we sought to provide a general characterization of the mechanisms of dissipation of phases described by Zeh, Zurek, and others, but in a way that would not require an artificial or poorly defined division of the closed system into subsystem and environment. For this reason we were led to notions of the decoherence of histories that imply consistency, of course, but are stronger and should not be confused with it.

The differences we have described should not obscure the fact that our work lies within the class of consistent histories formulations of the quantum mechanics of closed systems, but should also not obscure the fact that decoherence of histories, as we have defined it, is not the same as just consistency. 